\title{
Effect of Reducing the Number of Leads from Body Surface Potential Mapping in Computer Models of Atrial Arrhythmias
}

\author{
Victor G Marques $^{1}$, Miguel Rodrigo ${ }^{2}$, María S Guillem ${ }^{2}$, João Salinet ${ }^{1}$ \\ ${ }^{1}$ Federal University of ABC, São Bernardo do Campo, Brazil \\ 2 ITACA Institute, Universitat Politecnica de Valencia, Spain
}

\begin{abstract}
Body surface potential mapping (BSPM) can be an important tool in ablation therapy planning. Results obtained with high resolution (HR) computer models must be translated to realistic numbers of leads. This study aims to evaluate the impact on atrial tachycardia (AT), flutter (AFL) and fibrillation (AF) characterization by reducing the number of BSPM leads. 19 realistic computer simulations with 567 leads (HR) have been used to characterize the arrhythmias concerning the dominant frequencies (DF) and phase singularity point (SP) distributions. DF maps were generated combining Welch periodogram and activation detection with wavelet transform modulus maxima. Phase was calculated with Hilbert transform on signals filtered around the highest DF $( \pm 1 \mathrm{~Hz})$; dynamics of SPs were analyzed using histograms (heatmaps, HMs) and connecting SPs along time (filaments). The analyses were reproduced for 6 layouts with 252 to 16 leads and results were compared using the structural similarity index (SSIM), sensitivity and precision in SP detection and analyzing features extracted from the maps. SSIM was lower in AF than in AFL or AT for DF maps and HMs, but was in average above 0.6 for layouts with 32 leads or more. In HMs, a loss in spatial resolution with fewer leads is reflected in decreasing values for sensitivity and precision. Features from DF maps, filaments or HMs were statistically equivalent in all layouts.
\end{abstract}

\section{Introduction}

Supraventricular tachyarrhythmias, including focal atrial tachycardia (AT), atrial flutter (AFL) and atrial fibrillation (AF), are among the most common cardiac arrhythmias in the world and can pose serious risks, such as an increased chance of thromboembolic events [1].

These arrhythmias are driven by localized sources, which can be targeted in radio-frequency ablation therapy to restore sinus rhythm [1]: AT is maintained by ectopic foci. AFL is driven by a macro-reentrant circuit, localized typically, but not obligatorily, around the cavotricuspid isthmus. AF has been shown to be maintained by ectopic, re-entrant circuits and functional rotors, which appear preferably when structural and/or functional atrial remodeling occurs [2].

The detection of the driving mechanisms and their location is crucial to the success of ablation therapies [1]. Non-invasive analyses of the driving sources using body surface potential mapping (BSPM) might be important auxiliary tools in clinical practice before electrophysiological studies and catheter ablation, reducing procedural time and their associated risks [3].

Research based on computer models yields complementary information to that obtained with patients, giving a much deeper interpretation of experimental data [4]. However, the conclusions obtained with simulations need to be translated into realistic settings, where a limited volume of data is available.

This study aims to quantify the impact on the results of frequency and phase analyses on BSPM from atrial arrhythmias when reducing the large number of leads available in a model to realistic clinical settings.

\section{Methods}

The methodology is summarized in the block diagram in Fig. 1. All analyses were made in Python 3.6.

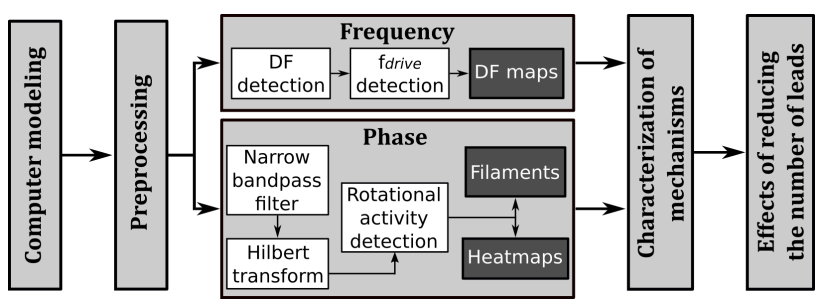

Figure 1. Block diagram of the project

\subsection{Computer models and preprocessing}

A realistic three-dimensional model of the atrial anatomy composed by 284,578 nodes and $1,353,783$ 
tetrahedrons ( $673.4 \pm 130.3 \mu \mathrm{m}$ between nodes) was used to simulate the electrical behavior of the atria in arrhythmic conditions. 19 simulations from arrhythmias caused by three distinct mechanisms were generated: AT (4) driven by an ectopic focus; AFL (4) driven by a macroreentrant circuit; AF (11) driven by functional rotors [5]. BSPMs were obtained by solving the forward problem with the boundary element method (sampling frequency $=500 \mathrm{~Hz}$ ), resulting in 771 data points, of which 567 were selected by excluding points inside the waist, neck, and arms (high resolution - HR).

Subsets of these points were selected to approximate lead layouts with different numbers of points: 252 [6], 131 [3], 67 [7], 64 [8], 32 and 16 (Fig. 2). The methods described below were applied for all the lead layouts and for the HR configuration for posterior comparison.

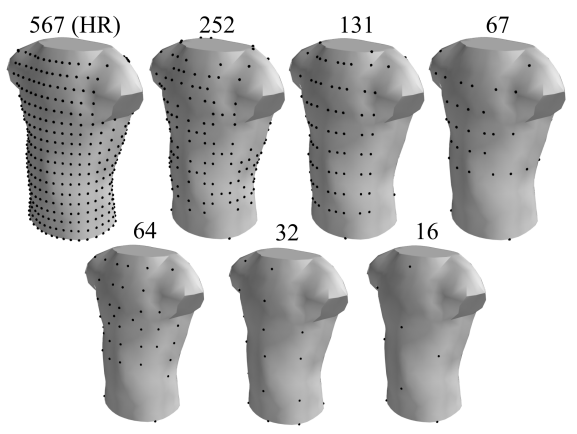

Figure 2. Lead layouts

Data points were reshaped into a 2D representation by projecting their positions onto a cylinder and unwrapping it. Signals were then interpolated into a $30 \times 65$ grid using cubic splines and the resulting data were bandpass filtered between 2 and $20 \mathrm{~Hz}$ (Butterworth, 4th order) [7].

\subsection{Frequency analysis}

The frequencies of the driver mechanisms ( $\left.f_{\text {drive }}\right)$ were estimated using a combination of analyses in time and frequency with spatial information from the BSPM.

Activation times were detected using a wavelet transform modulus maxima approach [9]. The average activation frequency was estimated as the inverse of the average interval between activation times. The spectral content for each data point was estimated via Welch periodograms (segments of $2.7 \mathrm{~s}$, Hanning window, zeropadded to 2048 points and with $90 \%$ overlap; frequency resolution of $0.37 \mathrm{~Hz}$ ). The dominant frequency (DF) of the spectrum was defined as the peak closest to the average activation frequency, thus reducing the detection of harmonics of the driver frequency.

The $f_{\text {drive }}$ was defined as the highest dominant frequency (HDF) on the torso after using a spatial mask ig- noring $2 \%$ of the highest DF values to avoid harmonics. The distributions of the DFs were analyzed by calculating the BSPM DF range (HDF-lowest DF) and identifying regions on the BSPM where $f_{\text {drive }}$ is expressed (i.e. connected portions of the torso with DF values in the range $f_{\text {drive }} \pm 0.2 \mathrm{~Hz}$ ). The average numbers and sizes of the regions were compared for every arrhythmia mechanism.

\subsection{Phase analysis}

BSPM signals were narrow bandpass filtered around $f_{\text {drive }}( \pm 1 \mathrm{~Hz}$, Butterworth, 4th order) [10] and were downsampled to $128 \mathrm{~Hz}$. Phase was determined using Hilbert transform [5].

Phase maps were generated for detecting singularity points (SPs), defined as the points around which all the phases converge [9], using a method based on edge detection: endpoints of edges detected by Canny's method were defined as SP candidates [11]. The detection of the SPs is based on the phase progression along 5 rings (radii from 2 to $10 \mathrm{~cm}$ ) around the SP candidates. Three criteria must be attended in at least two rings for a SP to be detected: the phase should progress in a range of at least $\pi$, the progression should be at least $60 \%$ ordered and there should be no phase leaps larger than $\pi$ [5].

The spatio-temporal distribution of the SPs was analyzed based on filament maps and heatmaps (HM). A filament is defined as the connection of the SPs in phase maps along subsequent time instants, around which at least one full cycle of rotation was sustained. Three-dimensional connected component analysis was applied using a kernel of shape 3x3x64 $(6.36 \mathrm{~cm}$ x 6.63 $\mathrm{cm} \times 0.5 \mathrm{~s}$ ) to connect and differentiate the individual filaments, with any two SPs belonging to the same region being assigned to the same filament. HMs are the histogram of the SPs belonging to filaments along time.

The number and duration of filaments were obtained for each model. Clusters of SPs in HMs were segmented with connected component analysis; the number, size, and density (\% of SPs in cluster/number of points in the cluster) of each cluster were calculated.

\subsection{Comparison between layouts}

Structural similarity index (SSIM, range 0 to 1) [12] was calculated between DF maps and HMs obtained with the different layouts to assess the changes in spatial information. Sensitivity and precision of SP detection were calculated considering the SPs detected with the HR configuration as the true locations. A $5 \times 5$ points window was used for both analyses (size: $11.0 \times 10.6 \mathrm{~cm}$ ).

Features extracted from the DF maps, filaments and heatmaps were statistically compared between layouts using the Friedman test (post-hoc: Nemenyi test). Sig- 
nificance was $p<0.05$; the null-hypothesis of the tests is that the measurements made with different layouts are compatible with each other.

\section{Results}

Figs $3 a$ and $3 b$ show the SSIM when comparing the DF maps and HMs obtained with the different layouts. SSIM mean values decrease as the number of leads is reduced, except for the 32 leads layoutIn the DF maps, AF presents the lowest values due to larger DF heterogeneity, in contrast to AT and AFL, where large regions present the same DFs. The similarities in the HMs are lower than in DF maps, especially for AT and AF. Fig. 4 shows examples of the SSIM for DF maps (a) and HMs (b).

Table 1 sums up the comparisons made between layouts, with the values obtained for each feature in the frequency and phase analyses, as well as the sensitivity and precision in SP detection. When extracting features from DF maps, no significant differences were encountered between the layouts, although large differences can be seen in individual models. Sensitivity and precision were above 0.7 for AFL with as few leads as 32 but decrease more clearly for AT and AF as fewer leads are used. However, when extracting features from the HMs, no significant differences were encountered between the layouts.

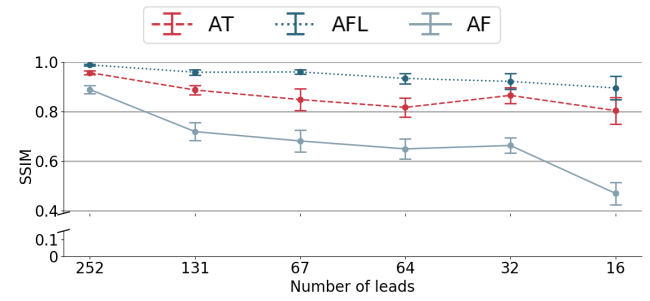

a) DF maps

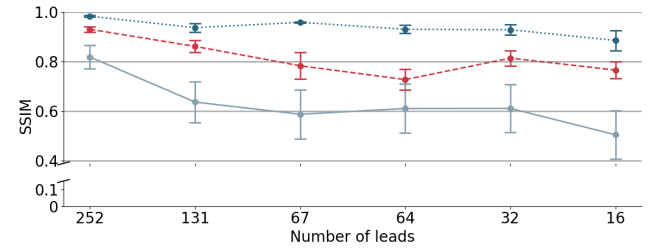

b) HMs

Figure 3. SSIM for DF maps and HMs (mean $\pm s e)$

\section{Discussion and Conclusions}

Several studies have been made in the past to determine an optimal BSPM setting, with lead numbers as limited as 8 [13]. A reduced number of leads is of interest for standardization of the BSPM method, the manufacturing of electrode vests and the reduction of the hardware requirements [13]. In this work, analyses in the frequency and phase domains, comprising many of the

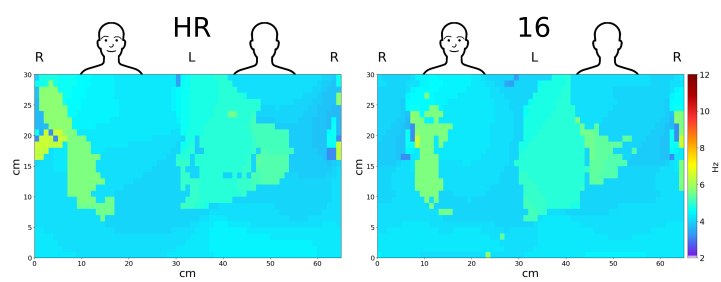

a) DF maps (AF in the left superior pulmonary vein, $S S I M=0.37$ )

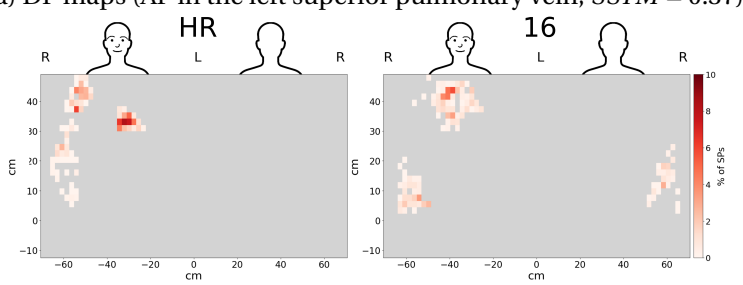

b) $\mathrm{HM}$ (AF in the right inferior pulmonary vein, $S S I M=0.72$ )

Figure 4. Examples of SSIM in DF maps (a) and HMs (b)

often used techniques for the processing of BSPM atrial signals, were used to assess the loss in spatial resolution with lead layouts ranging from 16 to 252 leads in comparison with a high resolution (567 leads) setting.

DF maps showed high similarity and overall patterns were observable in all lead layouts, especially for AT and AFL, which present more uniform DF distribution along the torso. The similarity is lower in AF, where more heterogeneity is present, even for higher numbers of leads. This results in larger differences in the features obtained with individual models, but on average all layouts were statistically equivalent.

Similar behavior is observed in the phase domain, where the AT and AFL filaments, more spatio-temporally stable than those from AF, result in very similar HMs, in contrast to those obtained with AF. High values in SSIM obtained for HMs may be due to regions without SPs, obscuring large spatial differences in the positions of the clusters. Reducing the number of leads affects intensely the sensitivity and precision in SP detection, reducing the ability of the BSPM to localize SPs in the phase domain. Nevertheless, the resulting filaments and HMs yield similar features in all layouts.

The results suggest that observations made in BSPMs obtained with a limited number of leads must be treated carefully, as spatial localization is reduced and large individual differences might appear in the frequency domain. However, several features extracted from DF maps and HMs are reliable and can be explored even with a very low number of leads.

\section{Acknowledgments}

This work was financed by the São Paulo Research Foundation (FAPESP), grant number 2017/19775-3. 
Table 1. Frequency (top) and phase (bottom) results obtained with the different layouts.

\begin{tabular}{|c|c|c|c|c|c|c|c|c|}
\hline & & HR & 252 leads & 131 leads & 67 leads & 64 leads & 32 leads & 16 leads \\
\hline \multicolumn{9}{|c|}{ Frequency Analysis } \\
\hline \multirow{3}{*}{$\begin{array}{c}f_{\text {drive }} \\
(\mathbf{H z})\end{array}$} & AT & $4.06 \pm 0.03$ & $4.06 \pm 0.03$ & $4.06 \pm 0.03$ & $5.04 \pm 0.97$ & $5.04 \pm 0.97$ & $4.06 \pm 0.03$ & $4.06 \pm 0.03$ \\
\hline & AFL & $4.09 \pm 0.12$ & $4.09 \pm 0.12$ & $4.09 \pm 0.12$ & $4.09 \pm 0.12$ & $4.09 \pm 0.12$ & $4.09 \pm 0.12$ & $4.09 \pm 0.12$ \\
\hline & $\mathbf{A F}$ & $5.58 \pm 0.23$ & $5.58 \pm 0.23$ & $5.58 \pm 0.23$ & $5.37 \pm 0.30$ & $5.58 \pm 0.23$ & $5.56 \pm 0.23$ & $5.59 \pm 0.22$ \\
\hline \multirow{3}{*}{ Range (Hz) } & AT & $0.76 \pm 0.70$ & $1.28 \pm 0.78$ & $1.10 \pm 0.69$ & $2.26 \pm 2.28$ & $1.89 \pm 1.61$ & $0.67 \pm 0.66$ & $0.70 \pm 0.67$ \\
\hline & AFL & $0.24 \pm 0.49$ & $0.24 \pm 0.49$ & $0.24 \pm 0.49$ & $0.24 \pm 0.49$ & $0.24 \pm 0.49$ & $0.24 \pm 0.49$ & $0.24 \pm 0.49$ \\
\hline & AF & $2.43 \pm 0.78$ & $2.42 \pm 0.78$ & $2.51 \pm 0.97$ & $2.23 \pm 0.94$ & $2.40 \pm 0.77$ & $2.42 \pm 0.81$ & $2.52 \pm 0.93$ \\
\hline \multirow{3}{*}{$\begin{array}{c}\text { number of } \\
f_{\text {drive }} \\
\text { regions }\end{array}$} & AT & $1.50 \pm 1.00$ & $1.5 \pm 1.00$ & $1.50 \pm 1.00$ & $2.75 \pm 2.06$ & $2.25 \pm 0.95$ & $2.50 \pm 3.00$ & $1.75 \pm 1.50$ \\
\hline & AFL & $1.00 \pm 0.00$ & $1.00 \pm 0.00$ & $1.00 \pm 0.00$ & $1.00 \pm 0.00$ & $1.00 \pm 0.00$ & $1.00 \pm 0.00$ & $1.25 \pm 0.25$ \\
\hline & $\mathbf{A F}$ & $2.64 \pm 0.43$ & $2.64 \pm 0.41$ & $3.64 \pm 0.59$ & $3.73 \pm 0.79$ & $3.09 \pm 0.53$ & $3.27 \pm 0.33$ & $4.45 \pm 0.43$ \\
\hline \multirow{3}{*}{$\begin{array}{l}\text { size of } f_{d r i v e} \\
\text { regions } \\
\text { (\% torso) }\end{array}$} & AT & $77.96 \pm 41.93$ & $78.03 \pm 42.04$ & $78.71 \pm 41.32$ & $52.37 \pm 54.67$ & $53.02 \pm 26.76$ & $76.04 \pm 46.28$ & $76.85 \pm 45.18$ \\
\hline & AFL & $97.62 \pm 2.1$ & $97.62 \pm 2.02$ & $97.29 \pm 2.34$ & $97.78 \pm 1.85$ & $96.81 \pm 2.79$ & $97.03 \pm 2.62$ & $74.77 \pm 24.91$ \\
\hline & $\mathbf{A F}$ & $22.27 \pm 10.63$ & $17.07 \pm 9.26$ & $10.76 \pm 7.60$ & $16.85 \pm 9.55$ & $11.21 \pm 8.28$ & $5.51 \pm 2.34$ & $3.37 \pm 1.22$ \\
\hline \multicolumn{9}{|c|}{ Phase analysis } \\
\hline \multirow{3}{*}{$\begin{array}{l}\text { Sensitivity in } \\
\text { SP detection }\end{array}$} & AT & - & $0.92 \pm 0.02$ & $0.86 \pm 0.03$ & $0.52 \pm 0.10$ & $0.43 \pm 0.08$ & $0.33 \pm 0.08$ & $0.11 \pm 0.02$ \\
\hline & AFL & - & $0.99 \pm 0.01$ & $0.85 \pm 0.07$ & $0.91 \pm 0.03$ & $0.71 \pm 0.07$ & $0.76 \pm 0.05$ & $0.16 \pm 0.06$ \\
\hline & $\mathbf{A F}$ & - & $0.83 \pm 0.02$ & $0.68 \pm 0.02$ & $0.48 \pm 0.03$ & $0.66 \pm 0.02$ & $0.51 \pm 0.02$ & $0.15 \pm$ \\
\hline \multirow{3}{*}{$\begin{array}{l}\text { Precision in } \\
\text { SP detection }\end{array}$} & AT & - & $0.91 \pm 0.03$ & $0.73 \pm 0.05$ & $0.53 \pm 0.11$ & $0.31 \pm 0.07$ & $0.25 \pm 0.04$ & $0.12 \pm 0.03$ \\
\hline & AFL & - & $0.95 \pm 0.03$ & $0.84 \pm 0.08$ & $0.94 \pm 0.03$ & $0.77 \pm 0.07$ & $0.71 \pm 0.06$ & $0.14 \pm 0.06$ \\
\hline & $\mathbf{A F}$ & - & $0.82 \pm 0.02$ & $0.62 \pm 0.02$ & $0.50 \pm 0.03$ & $0.62 \pm 0.02$ & $0.54 \pm 0.02$ & $0.17 \pm 0.01$ \\
\hline \multirow{3}{*}{ Filaments/s } & AT & $1.25 \pm 0.29$ & $1.25 \pm 0.33$ & $1.25 \pm 0.10$ & $1.25 \pm 0.18$ & $1.92 \pm 0.31$ & $1.44 \pm 0.45$ & $1.53 \pm 0.27$ \\
\hline & AFL & $0.96 \pm 0.31$ & $0.96 \pm 0.31$ & $0.97 \pm 0.31$ & $0.96 \pm$ & $1.21 \pm 0$ & $1.01 \pm 0.29$ & $0.98 \pm 0.51$ \\
\hline & $\mathbf{A F}$ & $1.16 \pm 0.20$ & $1.19 \pm 0.19$ & $1.22 \pm 0.18$ & $1.12 \pm 0.18$ & $1.15 \pm 0.17$ & $1.21 \pm 0.21$ & $1.62 \pm 0.23$ \\
\hline \multirow{3}{*}{$\begin{array}{l}\text { Filament } \\
\text { Duration } \\
\text { (\% signal) }\end{array}$} & AT & $78.42 \pm 13.8$ & $82.47 \pm 14.23$ & $89.93 \pm 7.70$ & $88.45 \pm 6.43$ & $79.79 \pm 9.5$ & $82.14 \pm 14.67$ & $69.02 \pm 10.14$ \\
\hline & AFL & $90.98 \pm 9.02$ & $90.94 \pm 9.06$ & $82.83 \pm 9.93$ & $90.45 \pm 9.55$ & $88.71 \pm 11.29$ & $91.58 \pm 8.36$ & $92.18 \pm 7.63$ \\
\hline & $\mathbf{A F}$ & $38.4 \pm 10.42$ & $38.79 \pm 10.39$ & $34.39 \pm 8.90$ & $34.06 \pm 7.76$ & $36.38 \pm 8.59$ & $34.17 \pm 8.36$ & $23.75 \pm 3.62$ \\
\hline \multirow{3}{*}{$\begin{array}{c}\text { Number of } \\
\text { HM } \\
\text { clusters }\end{array}$} & AT & $4.25 \pm 1.03$ & $4.25 \pm 1.44$ & $5.75 \pm 2.46$ & $5.50 \pm 2.53$ & $5.75 \pm 0.48$ & $4.5 \pm 1.04$ & $5.50 \pm 1.66$ \\
\hline & AFL & $2.00 \pm 0.00$ & $2.00 \pm 0.00$ & $2.50 \pm 0.29$ & $2.50 \pm 0.29$ & $3.50 \pm 1.19$ & $2.25 \pm 0.25$ & $3.00 \pm 1.22$ \\
\hline & $\mathbf{A F}$ & $10.64 \pm 2.11$ & $10.18 \pm 1.83$ & $9.91 \pm 1.99$ & $10.09 \pm 2.22$ & $12.45 \pm 2.63$ & $10.18 \pm 2.27$ & $11.73 \pm 1.98$ \\
\hline \multirow{3}{*}{$\begin{array}{c}\text { HM } \\
\text { cluster size } \\
\text { (\% torso) }\end{array}$} & AT & $0.64 \pm 0.14$ & $0.64 \pm 0.01$ & $0.63 \pm 0.07$ & $0.68 \pm 0.13$ & $0.72 \pm 0.10$ & $0.60 \pm 0.02$ & $0.47 \pm 0.07$ \\
\hline & AFL & $0.44 \pm 0.23$ & $0.47 \pm 0.22$ & $0.37 \pm 0.16$ & $0.35 \pm 0.09$ & $0.34 \pm 0.03$ & $0.56 \pm 0.33$ & $0.36 \pm 0.14$ \\
\hline & $\mathbf{A F}$ & $0.85 \pm 0.14$ & $0.94 \pm 0.16$ & $1.02 \pm 0.15$ & $1.17 \pm 0.24$ & $0.82 \pm 0.06$ & $0.91 \pm 0.12$ & $0.86 \pm 0.13$ \\
\hline \multirow{3}{*}{ SP density } & AT & $2.25 \pm 0.48$ & $3.16 \pm 1.54$ & $1.93 \pm 0.53$ & $2.13 \pm 0.55$ & $1.33 \pm 0.14$ & $2.49 \pm 0.73$ & $2.50 \pm 0.79$ \\
\hline & AFL & $10.79 \pm 2.71$ & $9.05 \pm 2.49$ & $9.06 \pm 2.61$ & $7.42 \pm 1.76$ & $7.57 \pm 2.52$ & $8.22 \pm 2.55$ & $14.76 \pm 7.44$ \\
\hline & $\mathbf{A F}$ & $0.62 \pm 0.11$ & $0.71 \pm 0.15$ & $0.77 \pm 0.17$ & $0.80 \pm 0.25$ & $0.60 \pm 0.11$ & $0.74 \pm 0.18$ & $0.63 \pm 0.20$ \\
\hline
\end{tabular}

\section{References}

[1] Issa ZF, et al. Clinical arrhythmology and electrophysiology: a companion to Braunwaldś heart disease. Elsevier Health Sciences, 2009.

[2] Narayan SM, et al. Treatment of atrial fibrillation by the ablation of localized sources: CONFIRM (conventional ablation for atrial fibrillation with or without focal impulse and rotor modulation) trial. J Am Coll Cardiol 2012; 60(7):628-636.

[3] Vanheusden FJ, et al. Systematic differences of noninvasive dominant frequency estimation compared to invasive dominant frequency estimation in atrial fibrillation. Comput Biol Med 2019;104:299-309.

[4] Dössel O, et al. Computational modeling of the human atrial anatomy and electrophysiology. Med Biol Eng Comput 2012;50(8):773-799.

[5] Rodrigo $\mathrm{M}$, et al. Technical considerations on phase mapping for identification of atrial reentrant activity in direct-and inverse-computed electrograms. Circulation Arrhythmia and Electrophysiology 2017;10(9):e005008.

[6] Haissaguerre M, et al. Driver domains in persistent atrial fibrillation. Circulation 2014;130(7):530-538.

[7] Guillem MS, et al. Noninvasive localization of maximal frequency sites of atrial fibrillation by body surface potential mapping. Circ Arrhythm Electrophysiol 2013; 6(2):294-301.

[8] Salinet J, et al. A 64-lead body surface potential mapping system. In 2017 Computing in Cardiology (CinC). IEEE, 2017; $1-4$.

[9] Vijayakumar R, et al. Methodology considerations in phase mapping of human cardiac arrhythmias. Circ Arrhythm Electrophysiol 2016;9(11):e004409.

[10] Rodrigo M, et al. Body surface localization of left and right atrial high-frequency rotors in atrial fibrillation patients: a clinical-computational study. Heart Rhythm 2014;11(9):1584-1591.

[11] Salinet J, et al. Propagation of meandering rotors surrounded by areas of high dominant frequency in persistent atrial fibrillation. Heart Rhythm 2017;14(9):12691278 .

[12] Wang Z, et al. Image quality assessment: from error visibility to structural similarity. IEEE transactions on image processing 2004;13(4):600-612.

[13] Rodrigo M, et al. Minimal configuration of body surface potential mapping for discrimination of left versus right dominant frequencies during atrial fibrillation. Pacing Clin Electrophysiol 2017;40(8):940-946.

Address for correspondence:

Victor Gonçalves Marques

Biomedical Engineering - CECS

Federal University of ABC

E-mail: vgmarques@ufabc.edu.br 